\title{
SUPPORTING INFORMATION Insight into OTFT Sensors Using Confocal Fluorescence Microscopy
}

\author{
Joshua N. Arthur, * Ajay K. Pandey, Jean-Michel Nunzi, Soniya D. Yambem* \\ Joshua N. Arthur \\ School of Chemistry and Physics, Faculty of Science, Queensland University of Technology (QUT), Brisbane, \\ QLD 4000, Australia \\ Centre for Materials Science, Queensland University of Technology (QUT), Brisbane, QLD 4000, Australia \\ E-mail: joshua.arthur@hdr.qut.edu.au \\ Dr. Ajay K. Pandey \\ School of Electrical Engineering and Robotics, Faculty of Engineering, Queensland University of Technology \\ (QUT), Brisbane, QLD 4000, Australia \\ Prof. Jean-Michel Nunzi \\ Department of Physics, Engineering Physics \& Astronomy, Queens University, Kingston, Ontario K7L 3N6, \\ Canada \\ Dr. Soniya D. Yambem \\ School of Chemistry and Physics, Faculty of Science, Queensland University of Technology (QUT), Brisbane, \\ QLD 4000, Australia \\ Centre for Materials Science, Queensland University of Technology (QUT), Brisbane, QLD 4000, Australia \\ E-mail: soniya.yambem@qut.edu.au
}

\section{Confocal setup details}

By default, the Nikon A1R confocal microscope used is configured with four different channels, corresponding to different sets of lasers and detectors paired with specific band pass filters. Each channel is optimised for exciting and detecting emission from a fluorescent dye commonly used in cell imaging (DAPI, FITC, TRITC, and Cy5). Both the TRITC and Cy5 channels are sensitive to P3HT photoluminescence, Cy5 being the most suitable of the available pre-set options, and therefore used in our initial experiments (for full HIFETs, sections 2.3-2.5). In theory, this is sub-optimal, due to poor alignment of the laser wavelength with the absorbance of P3HT (see Figure $2 \mathrm{~b}$ in main text). Additional data (for P3HT-only samples) were collected using the more optimal $561 \mathrm{~nm}$ laser (originally assigned to the TRITC channel), now paired with the detector from the Cy5 channel (bandpass filter centred on $700 \mathrm{~nm}, 75 \mathrm{~nm}$ bandwidth). After testing, we found both options give similar results. For the same laser power, the $561 \mathrm{~nm}$ yielded a stronger signal, but to avoid photobleaching the laser power had to be reduced and the final signal-to-noise ratio under either arrangement was similar.

To characterise variation in charge density in response to an analyte, a sequence of images was recorded. The initial image represents the device in an open circuit (or 'off') condition. A second image is taken with potentials applied to gate $\left(\mathrm{V}_{g}=-0.3 \mathrm{~V}\right)$ and drain $\left(\mathrm{V}_{\mathrm{ds}}=-1.0 \mathrm{~V}\right.$ ) electrodes, with respect to the source electrode at ground. This is the standard sensing configuration ('on' condition). After the analyte ( $5 \mu \mathrm{L}$ of either deionised water or 30 wt. $\% \mathrm{H}_{2} \mathrm{O}_{2}$ ) is deposited, the sample is imaged immediately (<30 seconds) after deposition and then after 2 minutes. Finally, the 
device is returned to an open circuit condition, and an image is taken after 60 seconds. Image sequences were also recorded while keeping the device voltages 'off' while depositing the analyte.

Image processing involved first rotating the image to improve the alignment of the channel with the $y$-axis of the image. The rotation algorithm in MATLAB uses nearestneighbour interpolation to assign pixel values. Gaussian smoothing is applied to reduce effect of digital noise, the loss of sharpness having no impact on the largescale patterns under investigation in this study. Photoluminescence intensity, represented by the greyscale value of each pixel, is then converted into values representing the percent by which any given pixel changes with respect to the corresponding pixel in a chosen reference image. This reference image is the first image in an image sequence. These new pixel values are then remapped to occupy a majority of the available range (contrast enhancement). The same adjustment is performed on all images within a single data set and is chosen so as to avoid 'clipping' artefacts (loss of data in bright or dark areas). The final pixel intensity values are plotted as a heat map, the values represented by a colour on a spectrum from dark blue $(-\Delta P L)$ to dark red $(+\Delta P L)$, the green mid-point representing $0 \%$ change.

Profile plots represent the same change in PL as depicted in the heat map figures, except each value represents the average of all pixels recorded along the vertical axis, for a given horizontal position. 


\section{Absolute PL images}

Below, Figures S1 through S7, plot 'absolute' PL measured by confocal fluorescence microscopy. The same data is shown in relative terms (as percent difference) in the main text. The figures here undergo the same processing as described above (rotation, Gaussian smoothing, etc.), but the pixel values are only adjusted for contrast (no subtraction), and thus shows the data essentially as seen through the microscope. The intensity scale is arbitrary (low to high $\mathrm{PL}$ ), and unique to each image sequence.

(a)
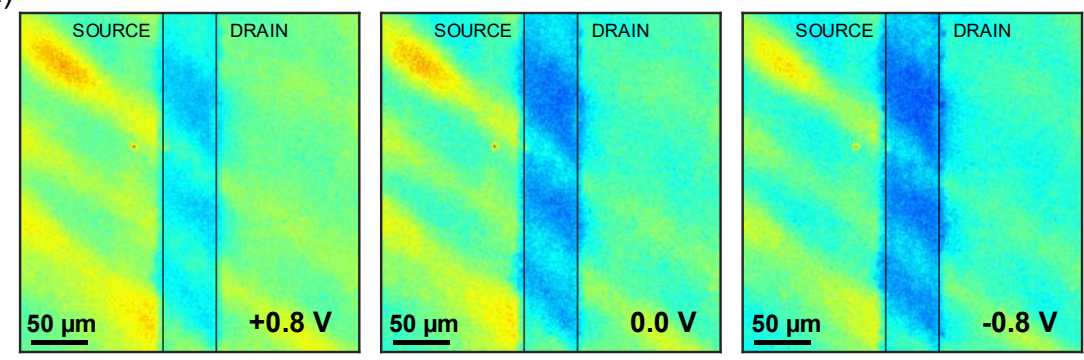

(b)
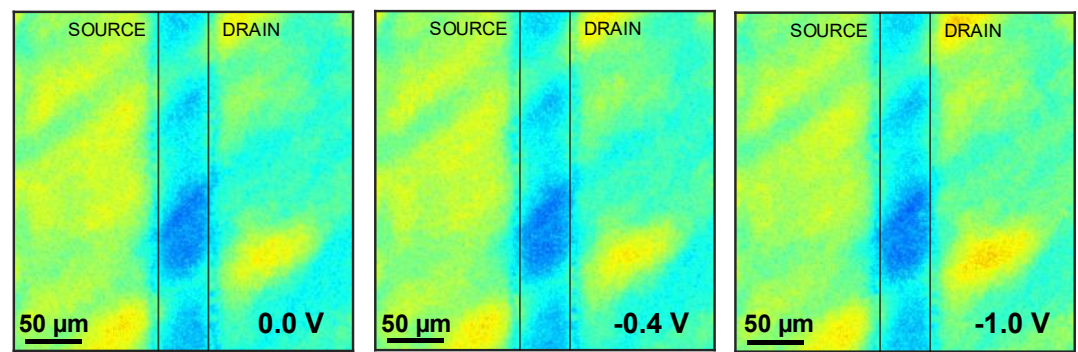

Figure S1: Confocal images showing absolute PL intensity, shown as relative PL in Figure $3 a$ and $3 c$ in the main text. (a) Effect of gate voltage, (b) effect of drain voltage.
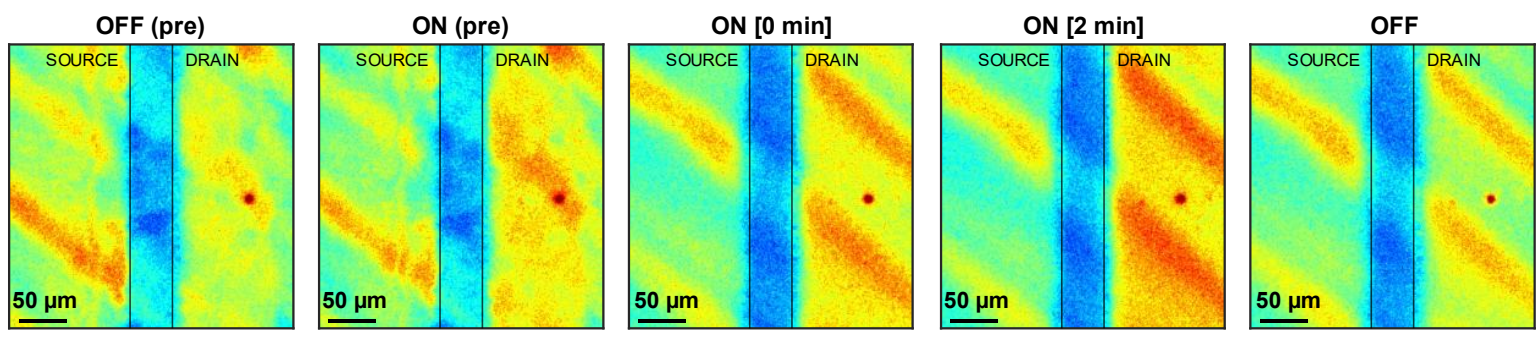

Low PL

High PL

Figure S2: Confocal images showing absolute PL intensity, shown as relative PL in Figures $4 \mathrm{~b}-\mathrm{e}$ in the main text. Images show a chronological sequence for a single device, off and on (pre-analyte) then after deposition of deionised water while on (after 0 and 2 minutes), then finally after turning the device off again. 

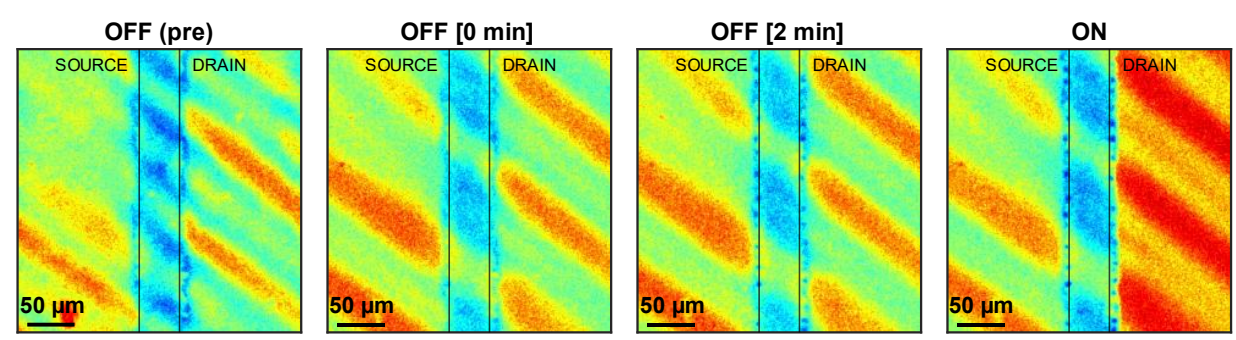

Low PL

High PL

Figure S3: Confocal images showing absolute PL intensity, shown as relative PL in Figures $5 b-d$ in the main text. Images show a chronological sequence for a single device, off (pre-analyte) then after deposition of deionised water while off (after 0 and 2 minutes), then finally after turning the device on.
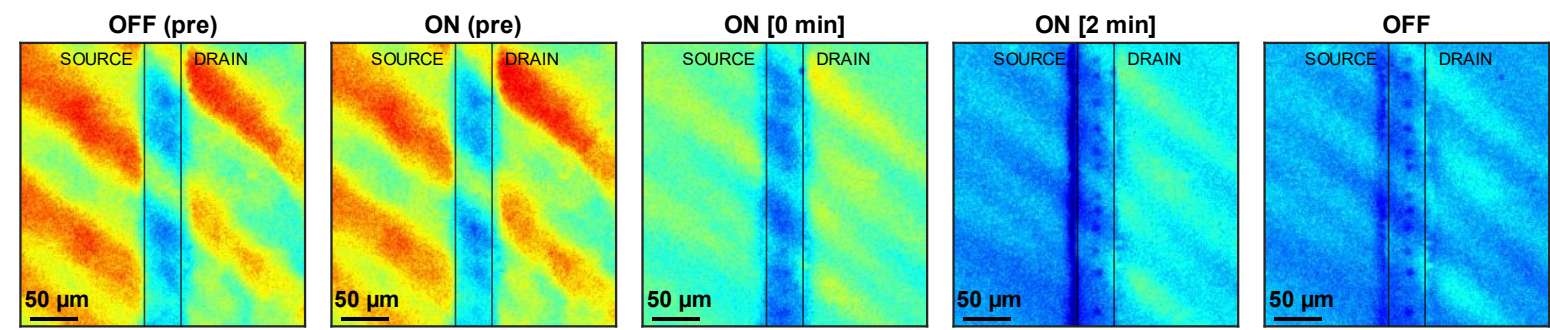

Low PL

High PL

Figure S4: Confocal images showing absolute PL intensity, shown as relative PL in Figures $6 b-e$ in the main text. Images show a chronological sequence for a single device, off and on (pre-analyte) then after deposition of hydrogen peroxide while on (after 0 and 2 minutes), then finally after turning the device off again.
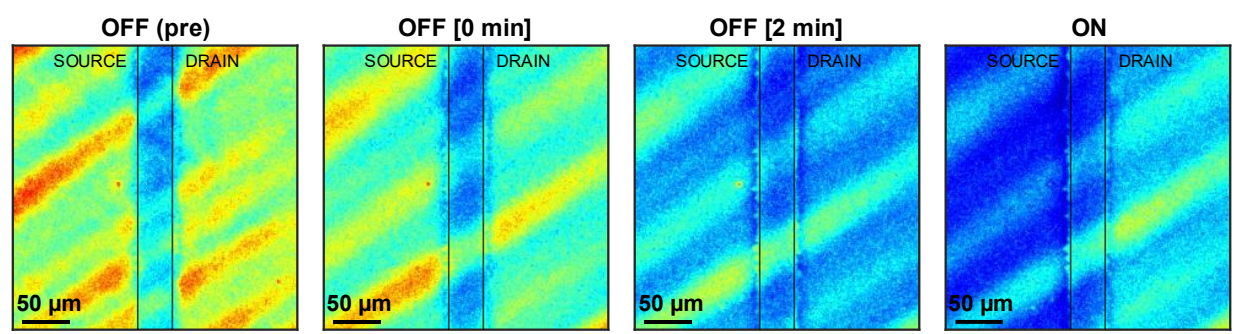

Low PL

High PL

Figure S5: Confocal images showing absolute PL intensity, shown as relative PL in Figures $7 \mathrm{~b}-\mathrm{d}$ in the main text. Images show a chronological sequence for a single device, off (pre-analyte) then after deposition of hydrogen peroxide while off (after 0 and 2 minutes), then finally after turning the device on. 

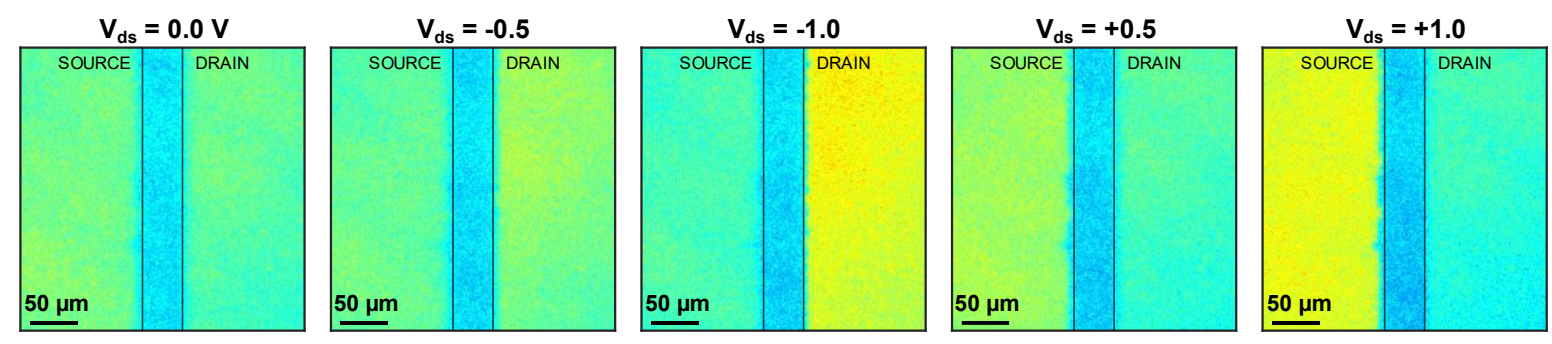

Low PL

High PL

Figure S6: Confocal images showing absolute $\mathrm{PL}$ intensity, shown as relative $\mathrm{PL}$ in Figures $8 \mathrm{~b}-\mathrm{e}$ in the main text. Images show a chronological sequence for a single $\mathrm{P} 3 \mathrm{HT}$ only device coated with deionised water, at different drain voltages.
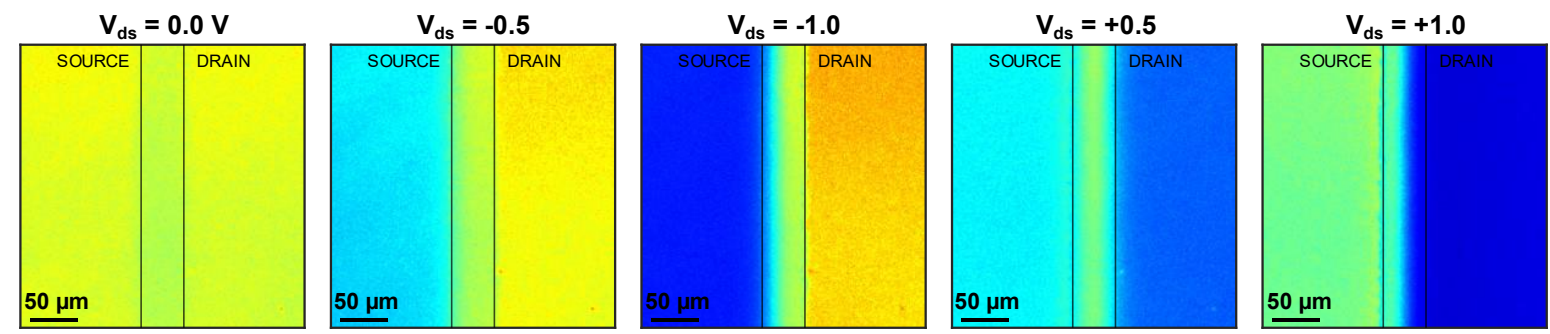

Low PL

High PL

Figure S7: Confocal images showing absolute PL intensity, shown as relative PL in Figures $9 \mathrm{~b}-\mathrm{e}$ in the main text. Images show a chronological sequence for a single $\mathrm{P} 3 \mathrm{HT}$ only device coated with hydrogen peroxide, at different drain voltages. 


\section{P3HT-only samples}
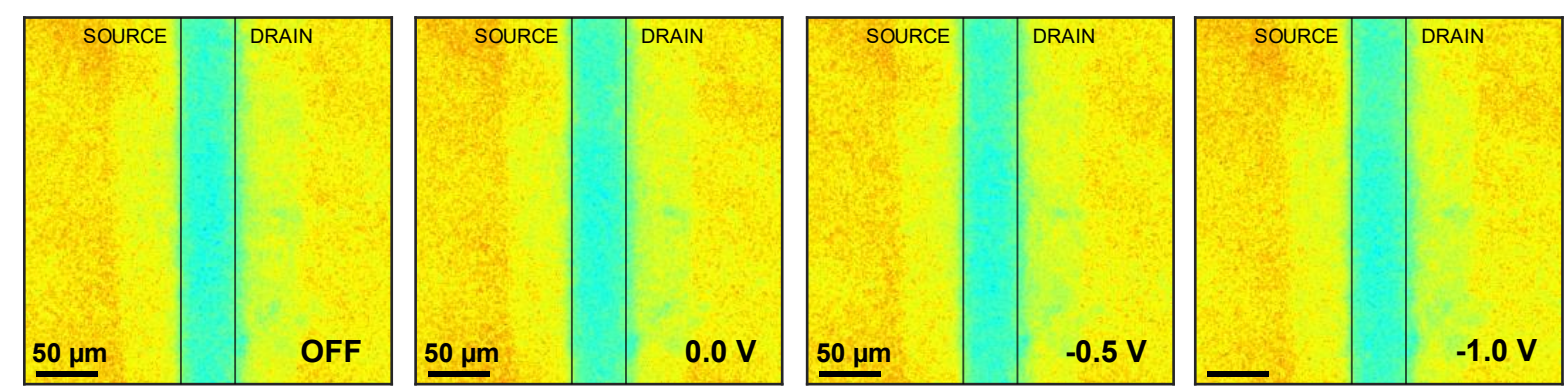

Low PL

High PL

(b)

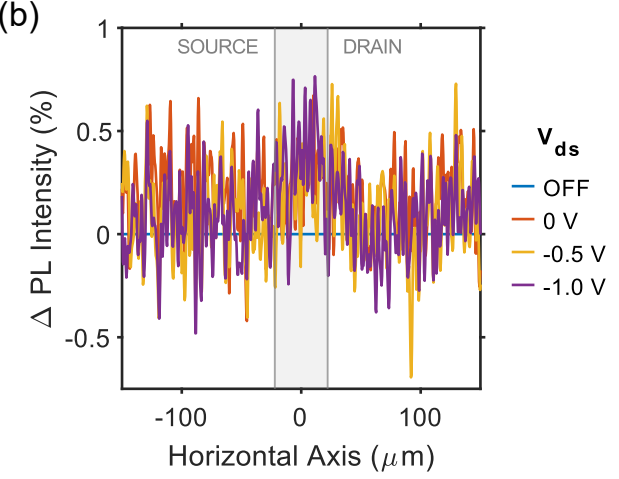

Figure S8: (a) Sequence of absolute confocal PL images of a pristine, dry P3HT-only sample. Drain voltage is varied. (b) Profile plot showing change in PL intensity for the same images, with respect to the 'off' state, along the horizontal axis. No significant change is observed with varied $V_{d s}$.

(a)

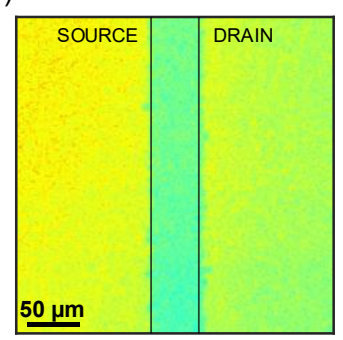

Low PL (b)

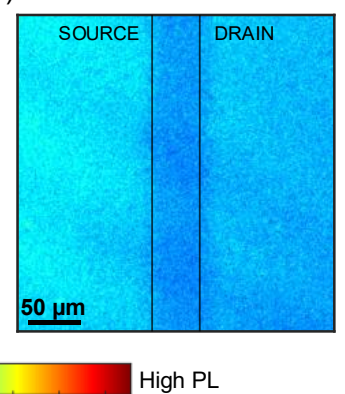

(c)

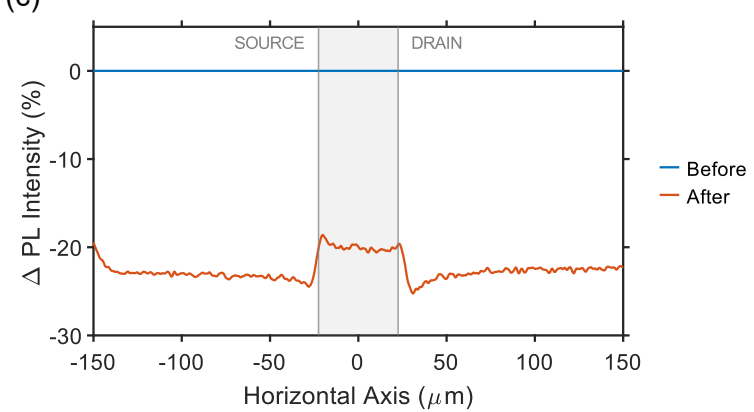

Figure S9: Absolute confocal PL images of a P3HT-only sample, (a) in pristine condition, and (b) after deposition of water. (c) The change in PL intensity, as a profile along the horizontal axis. 


\section{PL intensity profiles for alternative trials}

The main text shows one representative trial for each experimental condition, these selected from multiple trials on performed on separate devices. Here, we show the PL profile plots for each trial to demonstrate the differences between devices due to film thickness variations and the overall consistency and repeatability of the response to analytes tested.

\section{Effect of water: voltages on (Figure 4)}
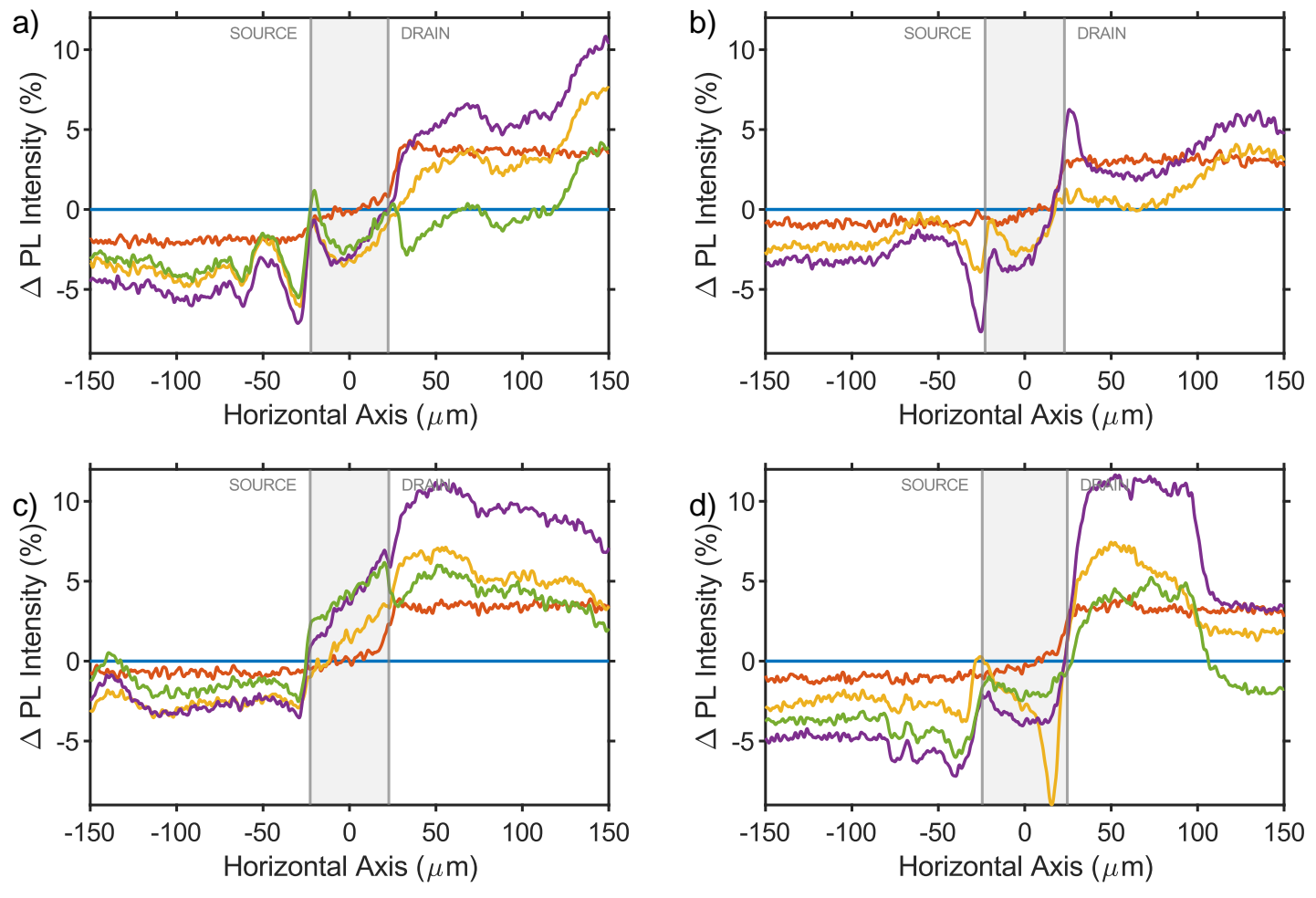

- OFF (pre) - ON (pre) - ON [0 min] - ON [2 min] - OFF

Figure S10: PL intensity profiles for HIFETs exposed to deionised water, while voltages are applied $\left(\mathrm{V}_{\mathrm{g}}=-0.3 \mathrm{~V}, \mathrm{~V}_{\mathrm{ds}}=-1 \mathrm{~V}\right)$. The same experiment is conducted on multiple samples. (a) Sample 1, the trial chosen to display in Figure 4 of the main text. (b) Sample 2. Data missing for final "OFF" state. (c) Sample 3. (d) Sample 4. 


\section{Effect of water: voltages off (Figure 5)}
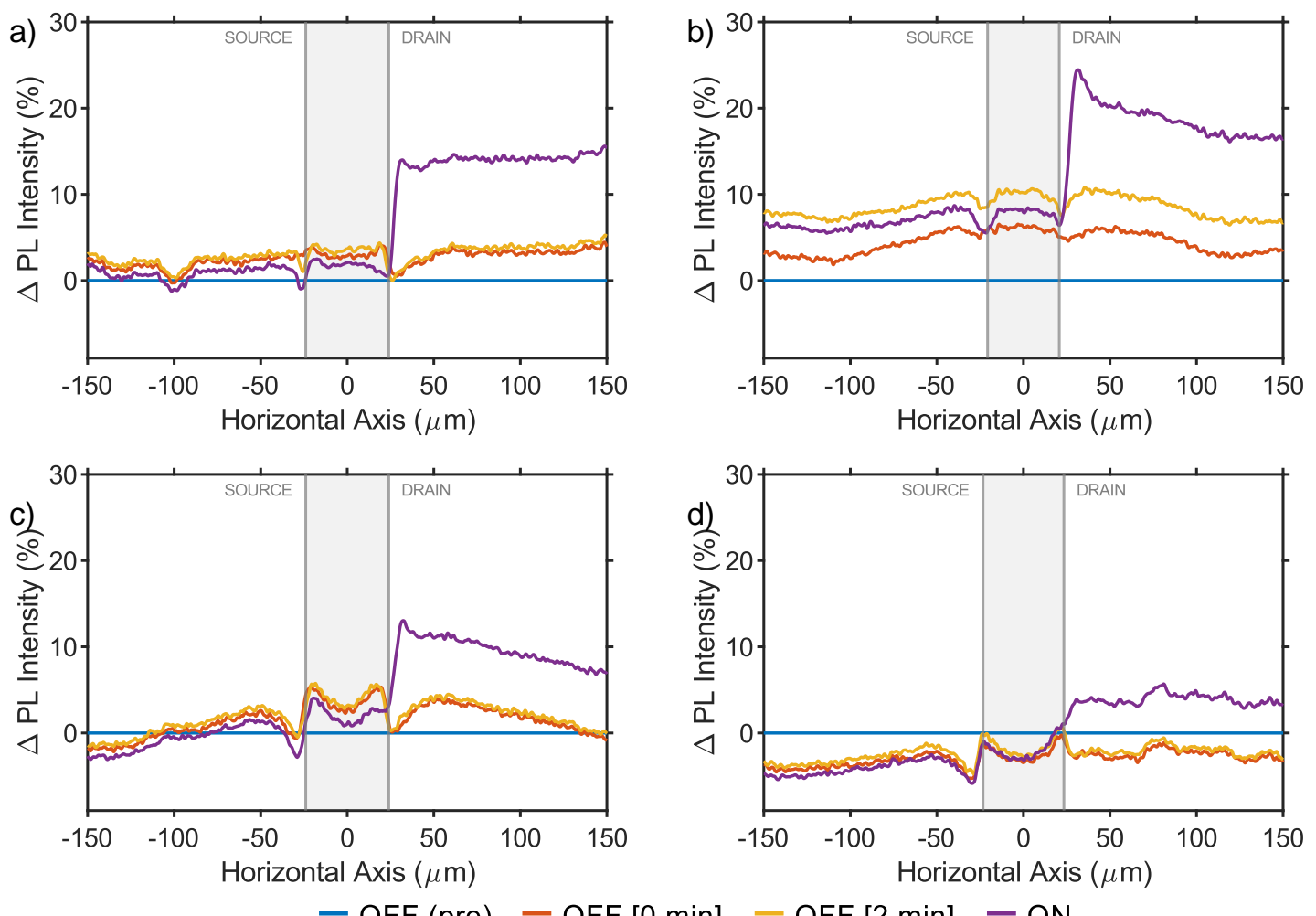

Figure S11: PL intensity profiles for HIFETs exposed to deionised water, while voltages are not applied. The same experiment is conducted on multiple samples. (a) Sample 1, the trial chosen to display in Figure 5 of the main text. (b) Sample 2. (c) Sample 3. (d) Sample 4. 


\section{Effect of $\mathrm{H}_{2} \mathrm{O}_{2}$ : voltages on (Figure 6)}
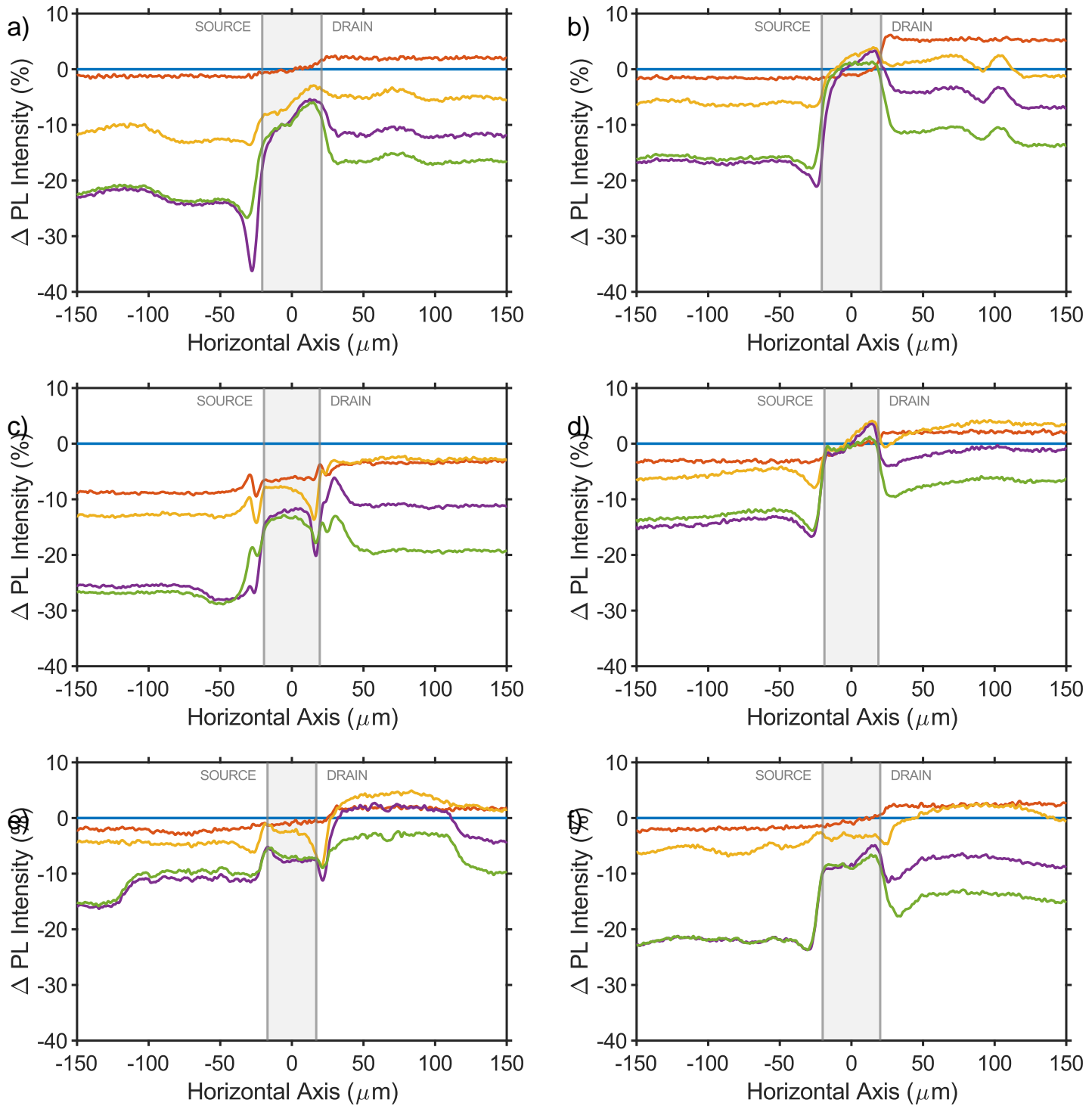

- OFF (pre) - ON (pre) - ON [0 min] - ON [2 min] - OFF

Figure S12: $\mathrm{PL}$ intensity profiles for HIFETs exposed to $\mathrm{H}_{2} \mathrm{O}_{2}$, while voltages are applied $\left(\mathrm{V}_{\mathrm{g}}=-0.3 \mathrm{~V}, \mathrm{~V}_{\mathrm{ds}}=-1 \mathrm{~V}\right)$. The same experiment is conducted on multiple samples. (a) Sample 1, the trial chosen to display in Figure 6 of the main text. (b) Sample 2. (c) Sample 3. (d) Sample 4. (e) Sample 5. (f) Sample 6. 


\section{Effect of $\mathrm{H}_{2} \mathrm{O}_{2}$ : voltages off (Figure 7)}
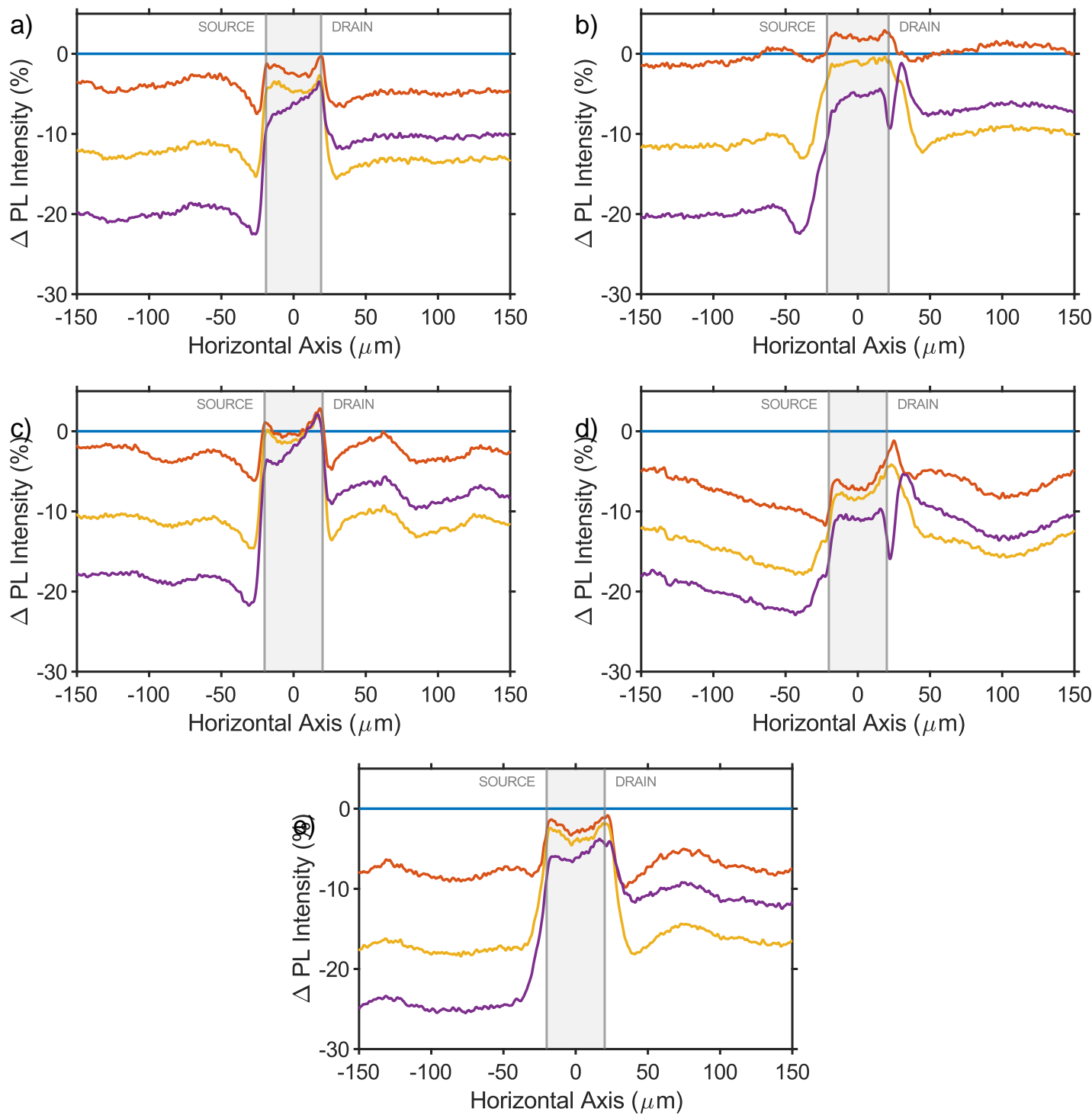

Figure S13: PL intensity profiles for HIFETs exposed to $\mathrm{H}_{2} \mathrm{O}_{2}$, while voltages are not applied. The same experiment is conducted on multiple samples. (a) Sample 1, the trial chosen to display in Figure 7 of the main text. (b) Sample 2. (c) Sample 3. (d) Sample 4. (e) Sample 5. 


\section{Example calculation: charge injected into channel}

PL quenching data can be used to estimate the number of holes being injected, using a straightforward calculation that begins with, (1) the density of molecules/monomers in the layer, (2) the proportion of molecules/monomers quenched, and (3) the number of molecules/monomers quenched per injected hole.

For the sake of having values for an initial approximation, we will assume that (3) for $\mathrm{P} 3 \mathrm{HT}$ is approximately that of $\alpha$-sexithiophene (molecular weight $=494 \mathrm{~g} \mathrm{~mol}^{-1}$ ), 200 molecules quenched per injected charge, as calculated by Fichou and Charra (1996). ${ }^{[1]}$ Rather than drawing a direct equivalence between the small molecule $\alpha-$ sexithiophene and the entire P3HT polymer, we will take the equivalent of a molecule that is quenched to be 8.7 thiophene monomers. Takeda \& Miller $(2021)^{[2]}$ determined that a positive polaron was delocalised over 8.7 thiophene units for a poly(3decylthiophene), a polymer similar to P3HT. Future work is required to confirm accurate quenching rates for $\mathrm{P} 3 \mathrm{HT}$.

With respect to (2) - the proportion of molecules quenched - this is derived from the $\mathrm{PL}$ quenching data. We assume that in the initial, pristine state, $100 \%$ of all $\mathrm{P} 3 \mathrm{HT}$ "units" (8.7 thiophene units) are emitting. If the PL decreases by $10 \%$, then we can say that $10 \%$ of those units are quenched. In reality, this is more complicated. One problem is that the P3HT is already doped in its "pristine" state, due to its exposure to PVP, air and moisture. So, we are not, in fact, starting from a state of $100 \%$ emission or $0 \%$ quenching. Another important point to note is that we do not measure PL intensity in absolute, quantitative terms, but as relative greyscale values in an image. This means values for percent change in $\mathrm{PL}$ are highly dependent on the baseline value, which is somewhat arbitrary (dependant on detector voltage, etc.). To make these calculations rigorous, proper calibration should be done. For the purpose of this demonstration, we will assume that our extracted $\Delta \mathrm{PL}$ values are representative of the true change in $\mathrm{PL}$ intensity.

\section{Density of P3HT monomers}

Given the P3HT thickness $(50 \mathrm{~nm})$, and the mass density $\left(1.1 \mathrm{~g} \mathrm{~cm}^{-3}\right)^{[3]}$ we can calculate the area density:

$$
\begin{aligned}
\text { Area density } & =\rho\left(\frac{g}{m^{3}}\right) \times t(m) \\
& =1.1 \times 10^{6} \times 50 \times 10^{-9}=0.055\left(\frac{g}{m^{2}}\right)
\end{aligned}
$$

Given the molecular weight of the P3HT monomer $\left(\mathrm{C}_{10} \mathrm{H}_{14} \mathrm{~S}\right)$ of $166.28 \mathrm{~g} \mathrm{~mol}^{-1}$, we find the number of grams per monomer:

$$
\begin{aligned}
\text { Monomer weight } & =M / N_{A} \\
& =166.28\left(\frac{g}{m o l}\right) \div 6.022 \times 10^{23}\left(\frac{\text { monomers }}{\text { mol }}\right) \\
& =2.761 \times 10^{-22}\left(\frac{g}{\text { monomer }}\right)
\end{aligned}
$$


Thus, we find the monomer area density:

$$
\begin{aligned}
\text { Monomer area density } & =\text { Area density }\left(\frac{g}{m^{2}}\right) \div \text { Monomer weight }\left(\frac{g}{\text { monomer }}\right) \\
& =0.055\left(\frac{g}{m^{2}}\right) \div 2.761 \times 10^{-22}\left(\frac{g}{\text { monomer }}\right) \\
& =1.99 \times 10^{20}\left(\frac{\text { monomers }}{m^{2}}\right)
\end{aligned}
$$

As we will be assuming that a "unit" that can be quenched is 8.7 monomers, we can simply divide this by 8.7 :

$$
\text { P3HT unit area densitity }=2.29 \times 10^{19}\left(\frac{P 3 H T^{\prime} \text { units }^{\prime}}{m^{2}}\right)
$$

\section{Holes injected into channel due to $\mathrm{H}_{2} \mathrm{O}_{2}$}

We find that the P3HT PL (apparently) decreases by $\sim 10 \%$ upon depositing $\mathrm{H}_{2} \mathrm{O}_{2}$. If $100 \%$ of the P3HT units were originally emitting, then $10 \%$ of those are now quenched, thus:

$$
\begin{aligned}
\text { Density of quenched monomers } & =\frac{10}{100} \times 2.29 \times 10^{19}\left(\frac{P 3 H T^{\prime} \text { units }^{\prime}}{m^{2}}\right) \\
& =2.29 \times 10^{18}\left(\frac{P 3 H T^{\prime} \text { units }^{\prime}}{m^{2}}\right)
\end{aligned}
$$

And if we say 200 P3HT 'units' are quenched per injected hole, we calculate the density of injected holes:

$$
\begin{aligned}
\text { Injected hole density } & =2.29 \times 10^{18}\left(\frac{P 3 H T^{\prime} \text { units }^{\prime}}{m^{2}}\right) \div 200\left(\frac{P 3 H T^{\prime} \text { units }^{\prime}}{\text { hole }}\right) \\
& =1.15 \times 10^{16}\left(\frac{\text { holes }}{m^{2}}\right)
\end{aligned}
$$

The channel area between source and drain is $A=2 \mathrm{~mm} \times 50 \mu \mathrm{m}=10^{-7} \mathrm{~m}^{2}$

$$
\text { Number of injected holes }=10^{-7}\left(\mathrm{~m}^{2}\right) \times 1.15 \times 10^{16}\left(\frac{\text { holes }}{\mathrm{m}^{2}}\right)
$$

\section{Plausibility check}

We have previously measured the drain-source current $\left(\mathrm{l}_{\mathrm{ds}}\right)$ response to depositing $\mathrm{H}_{2} \mathrm{O}_{2}$ (see Figure 1d in main report). Using the change in current, we can estimate the number of injected holes via another route, to check whether our rough estimate above lies within a reasonable range.

In one example, we see a modulation from $1.76 \mu \mathrm{A}$ to $5.27 \mu \mathrm{A}(\Delta \mathrm{lds}=3.51 \mu \mathrm{A})$. 
We know that the additional charge in the channel is, $Q=I t$. We want to know how much charge is in the channel at any given time while lds is constant, so we need the time, $t$, taken for a hole to travel through the channel. We can estimate this from mobility, $\mu$, using $v=\mu E$. Mobility for P3HT in OTFTs can vary by multiple orders of magnitude, and this is the primary source of uncertainty in this calculation. One report gives the mobility for a P3HT/PVP OFET at $0.1 \mathrm{~cm}^{2} \mathrm{~V}^{-1} \mathrm{~s}^{-1} .{ }^{[4]}$ This is generally considered as a "high" mobility for $\mathrm{P} 3 \mathrm{HT},{ }^{[5,6]}$ so we will use it as an upper limit for mobility, giving us a lower limit for charge.

We use a uniform electric field $\left(E=\frac{\Delta V}{d}\right)$ where the voltage between source and drain is $1 \mathrm{~V}$ and the distance is $50 \mu \mathrm{m}=0.005 \mathrm{~cm}$.

$$
\begin{aligned}
v & =\mu E \\
& =\mu \frac{\Delta V}{d} \\
& =0.1\left(\frac{\mathrm{cm}^{2}}{V s}\right) \times \frac{1(\mathrm{~V})}{0.005(\mathrm{~cm})} \\
& =20\left(\frac{\mathrm{cm}}{\mathrm{s}}\right) \\
t & =\frac{d}{v} \\
& =\frac{0.005(\mathrm{~cm})}{20\left(\frac{\mathrm{cm}}{\mathrm{s}}\right)}=2.5 \times 10^{-4}(\mathrm{~s}) \\
Q & =I t \\
& =3.51 \times 10^{-6}\left(\frac{C}{s}\right) \times 2.5 \times 10^{-4}(\mathrm{~s}) \\
& =8.78 \times 10^{-10}(\mathrm{C})
\end{aligned}
$$

We know that 1 Coulomb is $6.24 \times 10^{18}$ elementary charges, so,

$$
\begin{aligned}
\text { Number of injected holes } & =6.24 \times 10^{18}\left(\frac{\text { holes }}{C}\right) \times 8.78 \times 10^{-10}(C) \\
& =5.48 \times 10^{9}(\text { holes })
\end{aligned}
$$

Thus, we would expect the number of injected holes to be $\geq 5 \times 10^{9}$ holes.

This estimate is within the same order of magnitude from the value calculated using the PL quenching data, suggesting that our example calculation is in a reasonable range. 


\section{References}

[1] D. Fichou, F. Charra, Synth. Met. 1996, 76, 11.

[2] N. Takeda, J. R. Miller, J. Phys. Chem. B 2012, 116, 14715-14723.

[3] Y. Sun, Y. Han, J. Liu, Chinese Sci. Bull. 2013, 58, 2767.

[4] F.-Y. Yang, K.-J. Chang, M.-Y. Hsu, C.-C. Liu, J. Mater. Chem. 2008, 18, 5927.

[5] H. Sirringhaus, N. Tessler, R. H. Friend, Science 1998, 280, 1741-1744.

[6] L. Janasz, D. Chlebosz, M. Gradzka, W. Zajaczkowski, T. Marszalek, K. Müllen, J. Ulanski, A. Kiersnowski, W. Pisula, J. Mater. Chem. C 2016, 4, 11488-11498. 